\title{
Rendimiento y valor nutricional de avena asociada con vicia en condiciones altoandinas de Junín, Perú
}

\section{Marcial Enciso Altamirano ${ }^{1}$ - Carlos Gómez Bravo ${ }^{2}$ - Mariano Echevarría Rojas ${ }^{3}$ - César Osorio Zavala ${ }^{4}$ Orlando Chipana Quispe ${ }^{5}$ - Wilfredo Ruiz Camacho ${ }^{6}$}

Recepción: 24/05/2019 Aceptación: 02/07/2019

\section{Resumen}

En el presente estudio se plantea como objetivo determinar el rendimiento en materia seca y el valor nutricional de la asociación de avena-vicia, empleando diferentes variedades y proporciones en la siembra en condiciones altoandinas de la región Junín, Perú.

Se sembró la avena (Avena sativa), variedad Mantaro 15 y Centenario, asociada con vicia (Vicia sativa) en dos proporciones de siembra: de 80-20 y 70-30 Kg/ha en un diseño experimental de bloques completamente al azar; con arreglo factorial $2 \times 2$. Resultado: el rendimiento de la variedad Centenario de $15,833^{\mathrm{a}} \mathrm{Kg} / \mathrm{ha}$ de materia seca y variedad Mantaro $15 \mathrm{de} 12,765^{\mathrm{b}} \mathrm{Kg} /$ ha de materia seca; y en porcentaje de proteína de $6.5^{\mathrm{b}}$ y $7.9^{\mathrm{a}}$, respectivamente.

Se concluyó en rendimiento de materia seca $(\mathrm{Kg} / \mathrm{ha})$ y energía metabolizable $(\mathrm{MJ} / \mathrm{ha})$, la variedad Centenariovicia fue superior a Mantaro 15-vicia; y en contenido de porcentaje de proteína, la variedad Mantaro 15-vicia fue mejor a la variedad Centenario-vicia.

Palabras clave: Cultivo, avena-vicia, rendimiento, contenido nutricional.

\section{Abstract}

In the present objective is to determine the yield in dry matter and the nutritional value of the association of oatsvicia, using different varieties and proportions in the sowing in high Andean conditions of the Junín region, Peru.

Oats (Sativa Oat), Mantaro variety 15 and Centenary, associated with vicia (Vicia sativa), were sown in the sowing proportions: 80-20 and 70-30 Kg/ha in an experimental design of completely random blocks (DBCA); according to factorial $2 \times 2$. Result: the yield of the Centenary variety of $15.833^{\text {a }}$ to $\mathrm{Kg} / \mathrm{ha}$ of MS and Mantaro variety 15 of $12.765^{\mathrm{b}} \mathrm{Kg} / \mathrm{ha}$ of MS; and in percentage of protein of $6.5^{\mathrm{b}}$ and $7.9^{\mathrm{a}}$, respectively.

It was concluded in yield of dry matter ( $\mathrm{Kg} / \mathrm{ha})$ and metabolizable energy $(\mathrm{Mj} / \mathrm{ha})$, the variety centennial-Vice was superior to Mantaro 15- Vice; and in content of percentage of protein, the variety Mantaro 15-Vice was better to the variety Centennial-Vice.

Key words: Cultivation, oat-vine, yield, nutritional content.

1. Candidato PhD. del Programa de Doctorado en Nutrición, Estudiante de la Universidad Nacional Agraria La Molina, Lima-Perú; e-mail: encisoalt@gmail.com

2. PhD. Especialidad en Nutrición Animal, Profesor Principal, Departamento de Nutrición, Facultad de Zootecnia, Universidad Agraria la Molina (UNALM), Lima-Perú; e-mail: cagomez@lamolina.edu.pe

3. PhD. Especialidad en Nutrición Animal, Profesor Principal, Departamento de Nutrición, Facultad de Zootecnia, Universidad Agraria la Molina (UNALM), Lima-Perú; e-mail: mechevarria@lamolina.edu.pe

4. Ingeniero Zootecnista, Coordinador del Área de Ganadería INIA, Lima Perú, e-mail: cosorio@inia.gob.pe

5. Ingeniero Zootecnista, Magister of Science en Producción Animal, Especialista del Área de Estudios Socioeconómicos y Prospectiva Agraria, Instituto Nacional de Innovación Agraria, Lima-Perú; email: ochipana@inia.gob.pe

6. Ingeniero Agrónomo, Magister of Science en Producción Agrícola, Candidato PhD. Del Programa Doctorado en Agricultura Sustentable de la Universidad Nacional Agraria La Molina, Lima-Perú. Catedrático en la Universidad Nacional de Jaén; e-mail: wilfredo.agronomo@gmail.com 


\section{Introducción}

El cultivo en asociación permite obtener mayor cantidad y calidad de forraje, mejora el aporte de nutrientes, palatabilidad y digestibilidad, debido a que la avena proporciona energía; mientras la vicia proporciona principalmente proteína (INCALAC, 1997). La avena y vicia son plantas anuales pudiendo establecerse de 3,000 a 4,000 m.s.n.m. La vicia es una planta (semi trepadora y posee zarcillos) que se siembra generalmente asociado a un cereal para impedir su deterioro al contacto con el suelo (INIA, 2013).

En el cultivo en asociación de avena-vicia es importante conocer el momento oportuno de cosecha para aprovechar al máximo su rendimiento y contenido nutricional. Para usar como heno es recomendable la cosecha en evento fenológico al 100\% de floración hasta grano lechoso de avena; y de 50\% a más de floración de vicia. En este periodo, la avena tiene mayor contenido de energía y la vicia mayor aporte de proteína (INCALAC, 1997 y Flores et al., 2016).

Se ha realizado un estudio con la variedad Mantaro 15 asociada con Vicia sativa, con el objetivo de determinar el rendimiento y valor nutricional de la avena; de esta asociada con vicia y sus ensilajes en la zona altoandina del Perú: en condiciones de Pachacayo-
Huancayo, cosechados en evento fenológico: avena hoja bandera y botón floral de vicia, avena 100\% floración y 5-30\% floración de vicia; y avena grano leche y vicia mayor de $50 \%$ de floración fueron de 10.5, 16.5 y $17.5 \mathrm{tn} / \mathrm{ha}$ en materia $\mathrm{seca}^{7}$; mientras que el porcentaje de proteína cruda resultó de 10.9, 10.2 y 6.6, respectivamente (Enciso, 1998). Otro estudio para rendimiento de Avena sativa, variedad Mantaro 15 asociada con Vicia sativa, para condiciones de Pachacayo Jauja fue de $45.6 \mathrm{tn} /$ ha en materia verde (CESO PROYECT, 1996).

En otro estudio, Salcedo (2011) reportó que, la digestibilidad de Avena sativa en asociación con Vicia sativa en materia seca y de proteína cruda cosechados en evento fenológico al $100 \%$ de floración de avena, fueron de 59.9 y 76.9\%, respectivamente. La asociación de avena-vicia se puede utilizar en la alimentación del ganado en forma directa como forraje y también se puede conservar para épocas de escasez de alimento como heno, ensilado y pellets (INTA, 2014 y Sánchez, 2014). En el presente estudio se plantea como objetivo determinar el rendimiento $(\mathrm{Kg} / \mathrm{ha})$ y el valor nutricional de la asociación de avena-vicia, empleando diferentes variedades y proporciones en la siembra en condiciones altoandinas de la región Junín, Perú.

7. Este término también será expresado dentro del documento mediante su acrónimo MS. 


\section{Materiales y métodos}

Rendimiento y valor nutricional de avena asociada con vicia bajo condiciones de Junín. El estudio en la fase experimental se llevó a cabo en la Sociedad Agrícola de Interés Social Túpac Amaru en Pachacayo, ubicada a $220 \mathrm{~km}$ de carretera Lima a Huancayo, Provincia de Jauja, departamento de Junín; a una altitud de 3,600m.s.n.m., temperatura $8.6^{\circ} \mathrm{C}$ y precipitación anual de $730.6 \mathrm{~mm} /$ año.

\begin{tabular}{|l|c|}
\hline Tratamiento 1 & $\begin{array}{c}80 \text { Avena sativa var. Man- } \\
\text { taro15 + 20 Vicia sativa }\end{array}$ \\
\hline Tratamiento 2 & $\begin{array}{c}70 \text { Avena sativa var. Man- } \\
\text { taro15 + 30 Vicia sativa }\end{array}$ \\
\hline Tratamiento 3 & $\begin{array}{c}80 \text { Avena sativa var. Cen- } \\
\text { tenario + 20 Vicia sativa }\end{array}$ \\
\hline Tratamiento 4 & $\begin{array}{c}70 \text { Avena sativa var. Cen- } \\
\text { tenario + 30 Vicia sativa }\end{array}$ \\
\hline
\end{tabular}

Figura 1. Tratamientos (semilla $\mathrm{Kg} / \mathrm{ha}$ ).

\section{Instalación y Manejo de Cultivo}

- Análisis de suelo (caracterización). Se tuvo en cuenta la conductibilidad eléctrica, carbonato, $\mathrm{pH}$, capacidad de intercambio catiónico (CIC), porcentaje de materia orgánica $(\mathrm{MO})$, nitrógeno $(\mathrm{N})$, fósforo $(\mathrm{P})$, potasio $(\mathrm{K})$, magnesio $(\mathrm{Mg})$ y aluminio (Al). Se realizó en Laboratorio de suelos, Universidad Nacional Agraria La Molina (UNALM) en octubre, 2016.

- Preparación de terreno. Se preparó el terreno con tractor y rastra.
- Diseño de parcelas. Se utilizaron 24 parcelas experimentales en la Sociedad Agrícola de Interés Social (SAIS), Túpac Amaru, Pachacayo- Junín (figura 2).

- Siembra. La siembra se realizó al voleo dependiendo a las condiciones climáticas.

- Deshierbo. El deshierbo se realizó en forma manual cuando fue necesario.

- Abonamiento. Se fertilizó con 80-60-60 de NPK/ha (sulfato de amonio, fosfato diamónico y cloruro de potasio). Se aplicó el sulfato de amonio a la mitad de la siembra, y la otra mitad en macollamiento de avena; mientras con fosfato diamónico y cloruro de potasio. Todo se fertilizó en momento de la siembra.

- Cosecha. Se cosechó en evento fenológico al 50\% de floración y 50\% de grano leche.

Evaluación de las características agronómicas. En las evaluaciones de las características agronómicas se tomaron los siguientes parámetros tales como: porcentaje de germinación de semilla, área foliar, número de hojas por planta y altura de la planta (ver figura 2).

Evaluación de rendimiento en materia verde y materia seca en (Kg/ha). Por cada unidad experimental se realizaron cinco cortes al ras del suelo de todas las plantas, cuya corona se encontraba dentro de $1 \mathrm{~m}^{2}$, se registró el peso fresco inicial; y de cada 
I

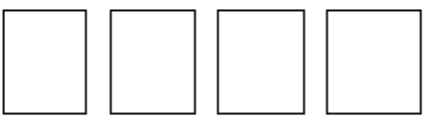

II
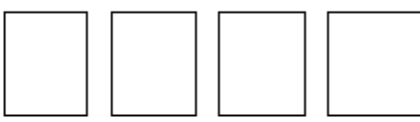

III
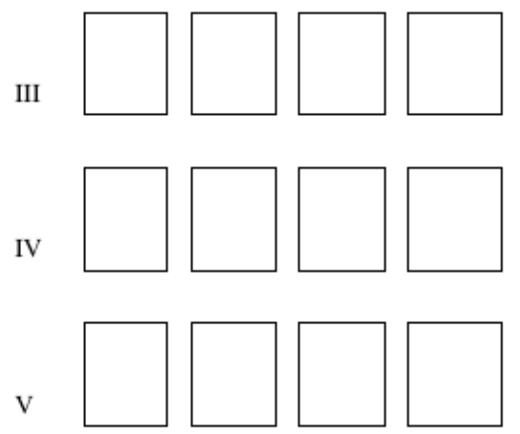

VI
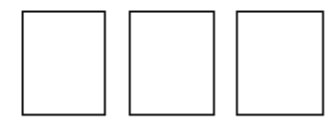

$375 \mathrm{~m}^{2}$

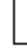

$25 \mathrm{~m}$

$105 \mathrm{~m}$

Figura 2. Croquis experimental en

Junín, Perú.

corte se tomó una sub muestra aleatoria. Las sub muestras fueron llevadas al Laboratorio de Evaluación Nutricional de Alimentos (LENA) de la Universidad Nacional Agria La Molina en mayo, 2017.

Las sub muestras fueron picadas y secadas a $60^{\circ} \mathrm{C}$ por 48 horas en una estufa de aire circulante; se pesaron las sub-muestras para obtener el peso final y así determinar el porcentaje de humedad inicial. Finalmente, se molieron en triturador Willey con una malla de $1 \mathrm{~mm}$ para el análisis químico, y se obtuvo la humedad final a $105^{\circ} \mathrm{C}$ por doce horas por Méto- do de Análisis Oficial Internacional (AOAC, 2005). Ahí se calculó la humedad total y materia seca para determinar el rendimiento de peso seco en $\mathrm{Kg} / \mathrm{ha}$.

La evaluación de rendimiento de materia verde de avena-vicia fue en el momento de cosecha (condiciones de campo); y la materia seca se llevó a cabo en el Laboratorio de Evaluación Nutricional de Alimentos de la Universidad Nacional Agraria La Molina, junio de 2017.

Composición química. La composición química de materia seca de los tratamientos (heno de avena-vicia) se realizó en el Laboratorio de Evaluación Nutricional de Alimentos de la Universidad Nacional Agraria La Molina, durante el mes de agosto, 2017 (AOC, 2005). Consistentes en:

- Proteína cruda

- Fibra detergente neutra (FDN $)^{8}$

- Digestibilidad aparente de la fibra detergente neutra a las 48 horas

- Digestibilidad aparente in vitro de materia orgánica (MO) a las 48 horas

- Calcio y Fósforo

Energía Metabolizable (EM). La energía metabolizable fue calculada y expresada en Mega Joul MJ/Kg y MJ/ha de forraje de avena-vicia de la siguiente manera:

a. Energía Metabolizable (MJ/Kg). Se calculó mediante la fórmula propuesta por Geenty y Rattray (1987). 
EM $(\mathbf{M J} / \mathrm{Kg})=0.16 \times$ Digestibilidad in $v i-$ tro de materia orgánica (DIVMO) ${ }^{9}$ expresado en MS.

b. Energía Metabolizable (MJ/ha). Se calculó el rendimiento de energía metabolizable de $1 \mathrm{Kg}$ forraje en MS y luego se multiplicó el rendimiento de forraje en MS por $10000 \mathrm{~m}^{2}$.

Diseño Experimental. El diseño experimental fue un diseño de bloques completamente al azar (DBCA) con arreglo factorial 2x2, dos variedades de avena (Mantaro 15 y Centenario) y dos proporciones (80kg avena - $20 \mathrm{~kg}$ de vicia y $70 \mathrm{~kg}$ avena - 30kg de vicia) de siembra, con seis bloques.

La comparación de medias se realizó mediante prueba de Tukey con un nivel de confianza de $(\mathrm{p}<0.05)$. El software estadístico utilizado fue el SAS.

\section{Resultados y Discusión}

Rendimiento y valor nutricional de avena asociada con vicia bajo condiciones de Junín.

\section{- Evaluaciones de las características agronómicas}

En la figura 3 se muestran el número de hojas por planta. La variedad Centenario fue superior a Mantaro 15, siendo relativamente inferior a los reportados por Noli y Ricapa (2009) y Montoya (2017); para la variedad Mantaro 15 de 4.5 y 4.6, respectivamente. Mientras para la variedad Centenario reportó un valor de 5.2 (Montoya, 2017). Los valores inferiores obtenidos en el presente estudio podrían deberse a la presencia de fenómenos naturales, tales como, sequías y heladas que aceleraron el secado de hojas (Carbonel, 2016).

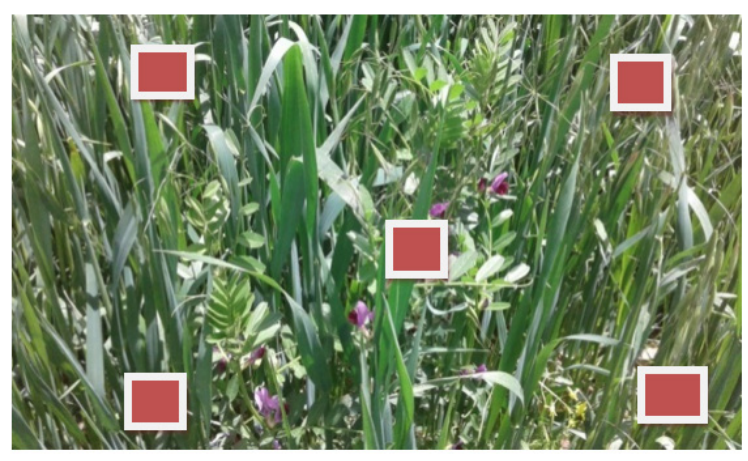

Figura 3. Planta de avena-vicia en $1 \mathrm{~m}^{2}$.

Los resultados de germinación de semilla de avena de la variedad Mantaro 15 y Centenario fueron de $93 \pm 1.1$ y $95 \pm 0.64$, correspondientemente; siendo similares a los valores reportados por Noli y Ricapa (2009; 92 y 93\%). Esta semejanza podría deberse a que las semillas fueron utilizadas de la misma zona (estación experimental agraria Santa Ana). Por otro lado, el porcentaje de germinación de semilla de Vicia sativa fue de $90 \pm 0.69 \%$.

En altura de planta fue superior la variedad Centenario a Mantaro 15. Sin embargo,

9. Dentro del documento también se hará relación a este término mediante su acrónimo. 
UNALM (2007) y Montoya (2017) reportaron valores para variedad Centenario de 1.95 y $1.22 \mathrm{~m}$, respectivamente, siendo superiores a lo obtenido en el presente estudio. Asimismo, en cuanto a la variedad Mantaro 15 se han reportado diferentes valores tales como, Bartl et al. (2007) quienes determinaron un valor inferior $(0.90 \mathrm{~m})$ a lo obtenido en el experimento; mientras que, Noli y Ricapa (2009) y Montoya (2017) obtuvieron valores superiores $(1.20$ y $1.15 \mathrm{~m})$ para dicha variedad. Esto evidente variación de los resultados, probablemente, atribuible a factores climáticos, zonas de ejecución y factor humano (Argote, 2005).

En área foliar, la variedad Centenario fue superior a Mantaro 15. Dicha superioridad podría estar relacionada a sus características agronómicas (UNALM, 2007): a mayor área foliar, mayor fotosíntesis (Galindo y Clavijo, 2007); y disminuye con aumento del estado vegetativo (Pérez et al., 2004). Mientras, la variedad Mantaro 15 -según lo reportado por Noli y Ricapa (2009)- presentan un periodo vegetativo corto, follaje verde intenso, tallo delgado y altura intermedia. Dichas características posiblemente influenciaron a valores inferiores frente a la variedad Centenario. Además, Montoya (2017) reportó de 90.1 y $105.7 \mathrm{~cm}^{2}$ para variedad Mantaro $15 \mathrm{y}$ Centenario, equitativamente, siendo inferiores al del presente estudio.
Los valores obtenidos empleando diferentes proporciones de siembra de avena-vicia (8020 y 70-30Kg/ha) de la variedad Mantaro 15 y Centenario fueron similares en número de hojas por planta, altura planta y área foliar en $\mathrm{cm}^{2}$ (ver tabla 1 ).

\section{Rendimiento en materia verde y materia $\operatorname{seca}(\mathrm{Kg} / \mathrm{ha})$}

En la tabla 2 se muestran los rendimientos en materia verde que fueron similares entre la variedad Mantaro 15 y Centenario. El rendimiento de materia verde de avena-vicia de la variedad Mantaro 15 fue superior al valor reportado por CESO PROYECT (1997) de 45.6tn/ha. Asimismo, Memoria Anual Zona Agraria X (1995) reportó valores para condiciones de la sierra central del Perú de 36.2 a $88.8 \mathrm{tn} / \mathrm{ha}$. Los resultados obtenidos del experimento se encuentran dentro de estos valores mencionados.

El rendimiento de forraje en materia seca $(\mathrm{Kg} /$ ha) entre las variedades fue superior estadísticamente $(\mathrm{P}<0.01)$ para la variedad Centenario en comparación a Mantaro 15; siendo estos resultados cercanos a lo reportado por INIA (2013) para dichas variedades (Mantaro 15 de: 14.0 y Centenario 14.5tn/ha). En el caso de la variedad Mantaro 15, lo reportado por Enciso (1998) fue de 16.5tn/ha, cosechado en evento fenológico al $100 \%$ de floración de avena, siendo ligeramente superior al valor obtenido en el estudio. Probablemente esto se 
Tabla 1. Evaluaciones agronómicas de avena

\begin{tabular}{|c|c|c|c|c|}
\cline { 2 - 5 } \multicolumn{1}{c|}{} & \multicolumn{2}{c|}{ Variedad de avena } & \multicolumn{2}{c|}{ Proporción de A/V } \\
\cline { 2 - 5 } & $\begin{array}{c}\text { Mantaro 15 } \\
\text { vicia }\end{array}$ & $\begin{array}{c}\text { Centenario }+ \\
\text { vicia }\end{array}$ & $\mathbf{8 0 - 2 0 K g / h a}$ & $\mathbf{7 0 - 3 0 K g / h a}$ \\
\hline Número de hoja/planta & $4.29^{\mathrm{b}} \pm 0.03$ & $4.99^{\mathrm{a}} \pm 0.07$ & $4.67^{\mathrm{a}}$ & $4.62^{\mathrm{a}}$ \\
\hline Altura planta m. & $1.0^{7 \mathrm{~b}} \pm 0.02$ & $1.16^{\mathrm{a}} \pm 0.01$ & $1.09^{\mathrm{a}}$ & $1.13^{\mathrm{a}}$ \\
\hline Área foliar cm & $144.13^{\mathrm{b}} \pm 4,46$ & $230.05^{\mathrm{a}} \pm 10.7$ & $183.68^{\mathrm{a}}$ & $190.50^{\mathrm{a}}$ \\
\hline
\end{tabular}

Proporción de A/V = Proporción de siembra de avena-vicia.

Tabla 2. Rendimiento en materia verde y materia seca $(\mathrm{Kg} / \mathrm{ha})$ de avena-vicia

\begin{tabular}{|c|c|c|c|c|}
\cline { 2 - 5 } \multicolumn{1}{c|}{} & \multicolumn{2}{c|}{ Variedad de avena } & \multicolumn{2}{c|}{ Proporción de A/V } \\
\cline { 2 - 5 } & $\begin{array}{c}\text { Mantaro 15 }+ \\
\text { vicia }\end{array}$ & $\begin{array}{c}\text { Centenario }+ \\
\text { vicia }\end{array}$ & $\mathbf{8 0 - 2 0 K g / h a}$ & $\mathbf{7 0 - 3 0 K g / h a}$ \\
\hline Materia Verde & $57,809^{\mathrm{a}} \pm 1,215$ & $63,342^{\mathrm{a}} \pm 2,669$ & $58,348^{\mathrm{a}}$ & $62,072^{\mathrm{a}}$ \\
\hline Materia Seca & $12,765^{\mathrm{b}} \pm 366$ & $15,833^{\mathrm{a}} \pm 639$ & $13,976^{\mathrm{a}}$ & $14,366^{\mathrm{a}}$ \\
\hline
\end{tabular}

Proporción de A/V = Proporción de siembra de avena-vicia.

a,b Superíndices con diferentes letras dentro de una misma fila indican diferencias significativas $(\mathrm{p}<0.05)$.

debe a la escasa precipitación y presencia de heladas en sitio de estudio que disminuyeron la producción (Carbonel, 2016).

Los rendimientos de materia verde y materia seca empleando diferentes proporciones de siembra de avena-vicia (80-20 y 70-30Kg/ha) de las variedades Mantaro 15 y Centenario fueron similares en condiciones de Junín. Sin embargo, la variedad Centenario fue superior en número hojas, altura planta y área foliar a Mantaro 15. Estas características agronómicas de la variedad Centenario posiblemente influyeron en alto rendimiento en materia seca (kg/ha) en comparación a Mantaro 15.
En la figura 4 se observa el análisis de interacción de rendimiento de materia seca $(\mathrm{Kg} / \mathrm{ha})$ de avena variedad Mantaro 15 y Centenario asociada con vicia $(80-20$ y $70-30 \mathrm{Kg} / \mathrm{ha})$ que no presentó interacción. No obstante, las variedades fueron diferentes, estadísticamente independiente a las proporciones de vicia que fue incluida; siendo superior la variedad Centenario en comparación a Mantaro 15.

\section{Composición química y energía metabolizable}

En la tabla 3 se aprecian que, la variedad Mantaro 15 fue superior $(\mathrm{P}<0.01)$ a Centenario en porcentaje de proteína cruda y fibra detergente neutra, resultando en porcentaje de 


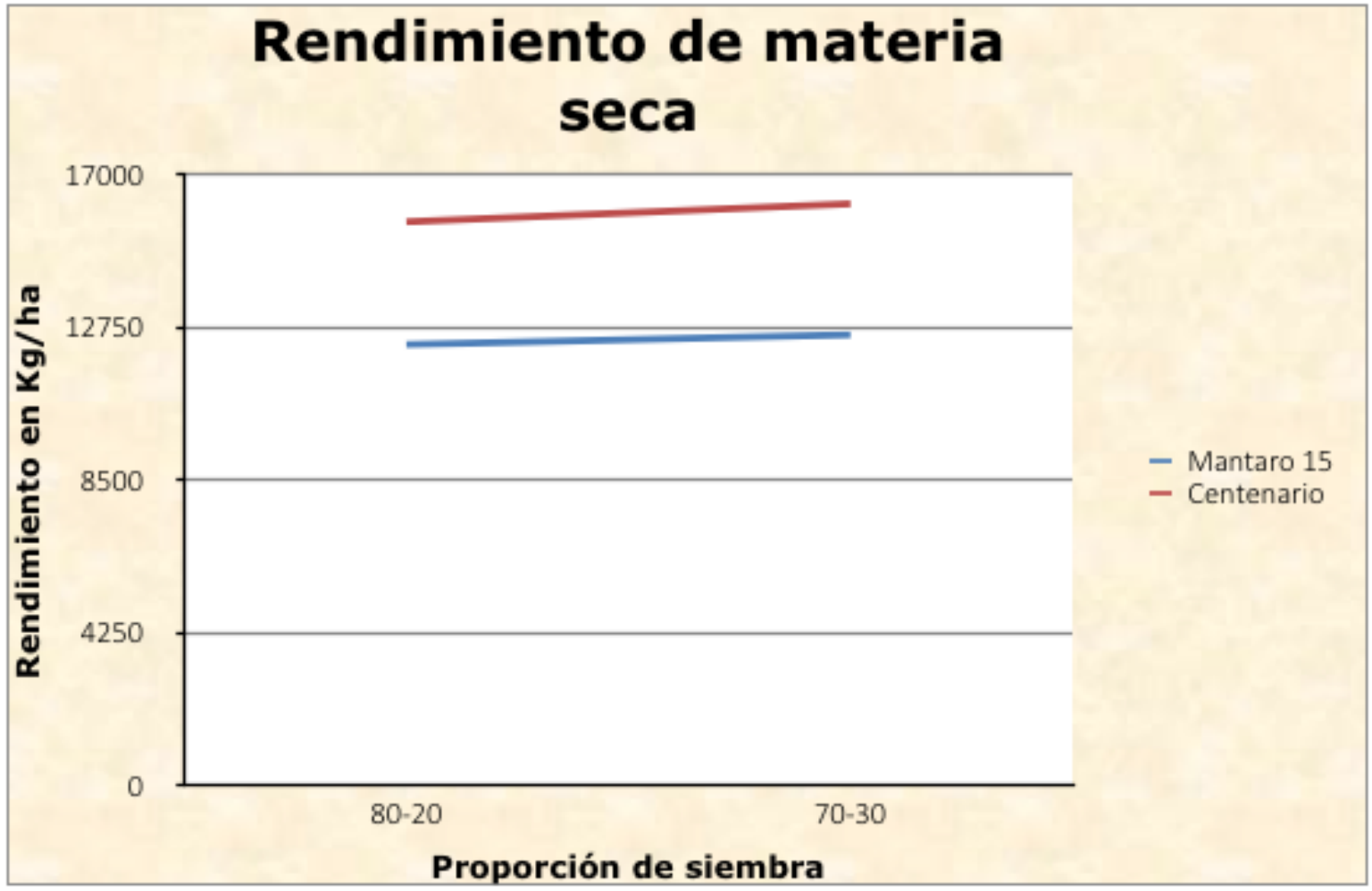

Figura 4. Interacción de rendimiento materia seca de avena-vicia.

proteína cruda inferior al valor reportado por Enciso (1998) de 10.2\%. Este bajo contenido de proteína cruda, está relacionado a la presencia de sequías y heladas registradas propias de la zona que afectaron la maduración de las plantas. Cabe señalar que la DIVMO fue superior en la variedad Centenario.

La energía metabolizable en $\mathrm{MJ} / \mathrm{Kg}$ y $\mathrm{MJ} / \mathrm{ha}$ resultó superior en la variedad Centenario $(\mathrm{P}<0.01)$ frente a Mantaro 15; este valor refleja el mayor rendimiento de forraje en materia seca de la variedad Centenario. Empleando diferentes proporciones de siembra de Avena-Vicia (80-20 y 70-30Kg/ha), el rendimien- to de $\mathrm{EM}(\mathrm{MJ} / \mathrm{Kg}$ y $\mathrm{MJ} / \mathrm{ha})$, contenido de proteína cruda, FDN y DIVMO fueron similares.

En figura 5 se muestra el análisis de interacción de proteína cruda de la variedad Mantaro 15 y Centenario asociada con vicia $(80-20$ y $70-30 \mathrm{Kg} / \mathrm{ha})$ que no presentó interacción. No obstante, fue superior en contenido de proteína la variedad Mantaro 15 comparada a Centenario independiente a las proporciones de siembra de avenavicia. Además, el contenido de proteína se incrementó con el aumento de proporción de vicia en ambas variedades. 
Tabla 3. Composición química y energía metabolizable de avena-vicia en base seca

\begin{tabular}{|c|c|c|c|c|}
\cline { 2 - 5 } \multicolumn{1}{c|}{} & \multicolumn{2}{c|}{ Variedad de avena } & \multicolumn{2}{c|}{ Proporción de A/V } \\
\cline { 2 - 5 } & $\begin{array}{c}\text { Mantaro 15 } \\
\text { vicia }\end{array}$ & $\begin{array}{c}\text { Centenario }+ \\
\text { vicia }\end{array}$ & $\mathbf{8 0 - 2 0 K g / h a}$ & $\mathbf{7 0 - 3 0 K g / h a ~}$ \\
\hline Proteína cruda (\%) & $7.9^{\mathrm{a}} \pm 0.2$ & $6.5^{\mathrm{b}} \pm 0.3$ & $6.8^{\mathrm{a}}$ & $7.6^{\mathrm{a}}$ \\
\hline FDN (\%) & $56.6^{\mathrm{a}} \pm 0.8$ & $50.1^{\mathrm{b}} \pm 0.7$ & $53.22^{\mathrm{a}}$ & $53.5^{\mathrm{a}}$ \\
\hline Ceniza (\%) & $6.2^{\mathrm{a}} \pm 0.1$ & $5.5^{\mathrm{b}} \pm 0.1$ & $5.70^{\mathrm{a}}$ & $5.9^{\mathrm{a}}$ \\
\hline DIVMO (\%) & $55.2^{\mathrm{b}} \pm 0.7$ & $60.7^{\mathrm{a}} \pm 0.6$ & $57.91^{\mathrm{a}}$ & $57.9^{\mathrm{a}}$ \\
\hline EM MJ/Kg & $8.9^{\mathrm{b}} \pm 0.1$ & $9.8^{\mathrm{a}} \pm 0.1$ & $9.23^{\mathrm{a}}$ & $9.3^{\mathrm{a}}$ \\
\hline & $109,747^{\mathrm{b}} \pm$ & $154,776^{\mathrm{a}} \pm$ & $130,118^{\mathrm{a}}$ & $134,405^{\mathrm{a}}$ \\
\hline
\end{tabular}

${ }^{\mathrm{a}, \mathrm{b}}$ Superíndices con diferentes letras dentro de una misma fila indican diferencias significativas $(\mathrm{p}<0.05)$.

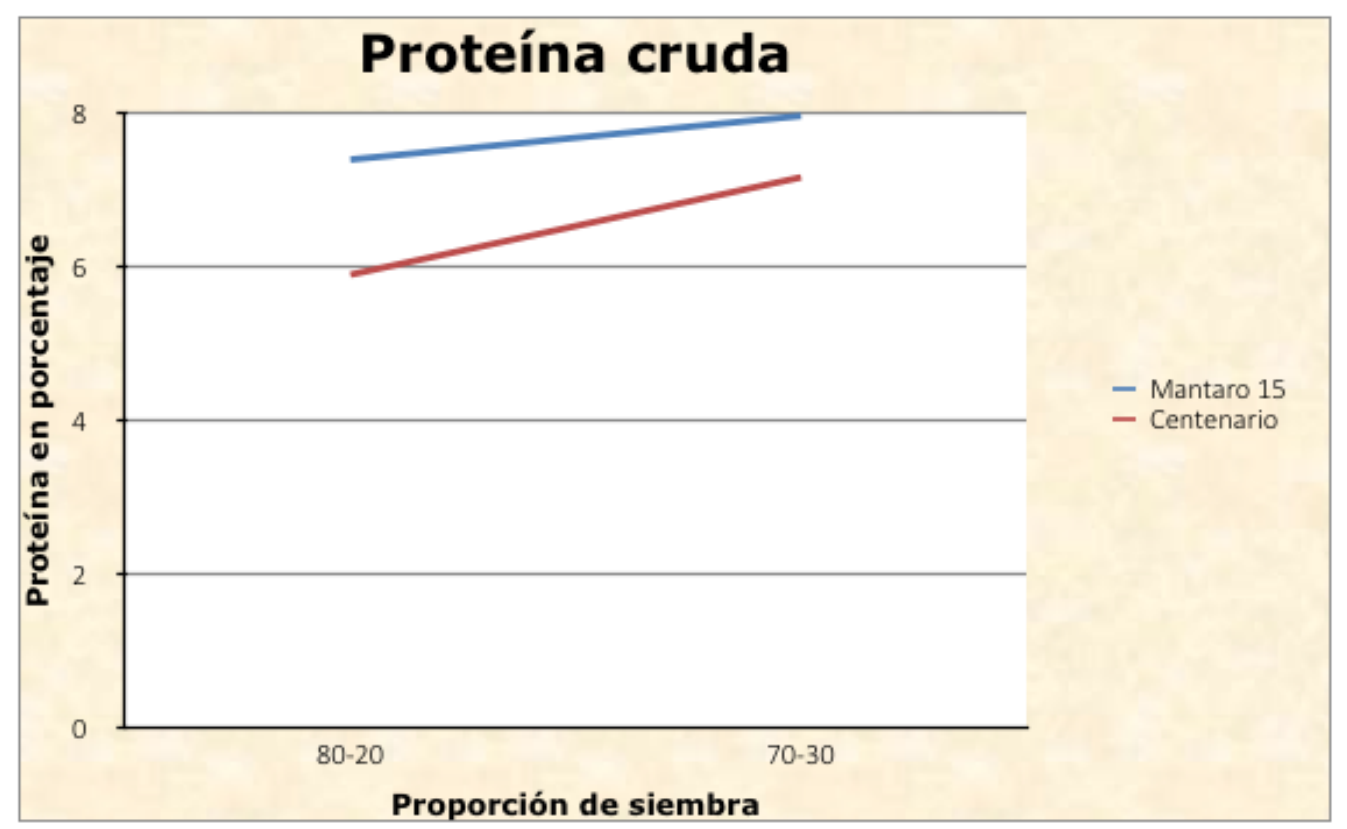

Figura 5. Interacción de proteína cruda de avena-vicia.

\section{Conclusiones}

La variedad Centenario-vicia fue superior a la variedad Mantaro 15-vicia, en rendimiento de en materia seca $(\mathrm{Kg} / \mathrm{ha})$ y energía metabolizable (MJ/ha). En contenido de proteína (\%), la variedad Mantaro 15-vicia fue mejor a la variedad Centenario-vicia. Para rendimiento de forraje, sembrar la variedad Centenario en asociación con vicia, para condiciones altoandinas de Junín, Perú. 


\section{Referencias}

Argote, G. (2005). Proyecto Pastos. Informe de resultados obtenidos de octubre del 2003 a junio del 2005. Proyecto PL 480- CTR 22. Ficha 35.

Association of Official Analytical Chemists (2005). International Official methods of analysis, 17 th edition.

Bartl, K.; Gómez, C.; Gamarra, J. y Hess, D. (2007). Potencial de especies forrajeras locales y mejoradas para la alimentación de ganado. Manual de extensión. Lima-Perú.

Carbonel, R. (2016). Caracterización de las Heladas Meteorológicas y Agronómicas e Impacto en alfalfa (Medicago sativa L.), avena (Avena sativa L.) y cebada (Hordeum vulgare L.) forrajeras de la región Puno. (Tesis de pregrado). Universidad Nacional Agraria La Molina, Lima- Perú

CESO Proyect (1996). Ceso International Service 175 Bloor Streets East Suit 400, South Tower, Toronto Canada.

Enciso, M. (1998). Valor Nutricional de Avena, Avena asociada con Vicia y sus Ensilajes en la Zona Altoandina del Perú (Tesis de maestría). Universidad Nacional Agraria La Molina, Lima-Perú. p. 111

Flores, J.; Sánchez, R.; Echevarría, F.; Gutiérrez, R.; Rosales, C. y Gonzáles, H. (2016). Producción y calidad de forraje en mezclas de veza común con cebada, avena y triticale en cuatro etapas fenológicas. Revista Mexicana de Ciencias Pecuarias, 7(3):275-291.

Galindo, J. y Clavijo, J. (2007). Modelos alométricos para estimar el área de los foliolos de arveja (Pisum sativum L.). Revista Corpoica - Ciencia y Tecnología Agropecuaria 8(1), 37-43. Recuperado de https://dialnet.unirioja.es > descarga > articulo

Geenty, G. y Rattray, V. (1987). The Energy Requirements of Grazing Sheep and Cattle. In livestock Feeding on Pasture. Occasional Publication, 10; 145 pp.

Industria Cajamarquina de Lácteos (enero, 1997). Boletín Agropecuario de Cajamarca. 
Instituto Nacional de Investigación Agraria (2013). Dirección de Investigación Agraria, Estación Experimental Agraria Santa Ana de Huancayo. Hoja divulgativa $\mathrm{N}^{\circ} 3-2013$. Recuperado de http://repositorio.inia.gob pe/handle/inia/159

Instituto Nacional de Investigación Agraria (2013). Dirección de Investigación Agraria, Programa Nacional de Investigación en Pastos y Forrajes de la Estación Experimental Baños del Inca Vicia INIA 906 Caxamarca. Folleto de divulgación Cajamarca, Perú.

Instituto Nacional de Investigación Agraria (2005). Dirección de General de Investigación Agraria Sub dirección de Investigación de crianzas. Proyecto de pastos y Forrajes. Estación Experimental Agraria Illpa-Puno.

Instituto Nacional de Tecnología Agropecuario (2014). 5ta. Jornada Nacional de forrajes conservados y recopilación de presentaciones técnicas. Estación Experimental Manfridi de Córdova, Argentina.

Kuehl, R. (2000). Diseño de experimentos, principios de diseño y análisis de investigación. Segunda edición. Edición Thomson y Learning, p. 666.

Memoria Anual Zona Agraria x (1995). Estación Experimental Santa Ana- Huancayo.

Montoya, K. (2017). Características agronómicas y valor nutricional de 7 cultivos forrajeros bajo secano en la sierra central. (Tesis de pregrado) Universidad Nacional Agraria La Molina, Perú; p. 88.

Noli, C. y Ricapa, F. (2009). Caracterización agronómica en avena forrajera en líneas promisorias para la producción de semilla en la sierra central del Perú. En: XXXII reunión científica anual de la asociación peruana de producción anual. Libro de resúmenes y programa oficial Tumbes Perú. Universidad Nacional de Tumbes Perú.

Pérez, J.; García, E.; Enríquez, J.; Quero, A. y Hernández, A. (2004). Análisis de crecimiento, área foliar específica y concentración de nitrógeno en hojas de pasto "mulato" (Brachiaria hibrido cv). Revista Mexicana de Ciencias Pecuarias. Recuperado de https://cienciaspecuarias.inifap.gob.mx/index.php/Pecuarias/article/view/1397 
Salcedo, G. (1998). Valor Nutritivo y Degradabilidad de Avena sativa y Vicia sativa. Pastos, 28 (1), 71-85. Recuperado de http://polired.upm.es/index.php/pastos/article/view/1241/1246

Sánchez, F. (2014). Módulo de Tecnologías de forrajes conservados. Programa Nacional de Agroindustria y agregado de valor INTA EEA Manfredi. Producción Animal Argentina.

Universidad Nacional Agraria La Molina (2007). Programa de investigación y proyección social en cereales y granos nativos. La Molina Lima Perú.

Wernli, C. y Ojeda, F. (1990). Metodología para investigaciones sobre conservación y utilización de los ensilajes. En Nutrición de los Rumiantes: Guía Metodológica de Investigación Ed. M. Ruiz, San José Costa Rica. 